\title{
Impaired endocytosis of the ion channel TRPM4 is associated with human progressive familial heart block type I
}

\author{
Martin Kruse, ${ }^{1}$ Eric Schulze-Bahr, ${ }^{2,3}$ Valerie Corfield, ${ }^{4}$ Alf Beckmann, ${ }^{1}$ Birgit Stallmeyer, ${ }^{2,3}$ \\ Güven Kurtbay,, ${ }^{1}$ Iris Ohmert, ${ }^{1}$ Ellen Schulze-Bahr, ${ }^{2,3}$ Paul Brink, ${ }^{4}$ and Olaf Pongs ${ }^{1}$ \\ ${ }^{1}$ Institut für Neurale Signalverarbeitung, Zentrum für Molekulare Neurobiologie, Universität Hamburg, Hamburg, Germany. \\ 2Institut für Genetik von Herzerkrankungen, Universitätsklinikum Münster, Münster, Germany. 3Interdisziplinäres Zentrum für Klinische Forschung, \\ Universität Münster, Münster, Germany. ${ }^{4}$ Department of Internal Medicine and Department of Biomedical Sciences, \\ Faculty of Health Sciences, University of Stellenbosch, Matieland, South Africa.
}

\begin{abstract}
Progressive familial heart block type I (PFHBI) is a progressive cardiac bundle branch disease in the His-Purkinje system that exhibits autosomal-dominant inheritance. In 3 branches of a large South African Afrikaner pedigree with an autosomal-dominant form of PFHBI, we identified the mutation c.19G $\rightarrow A$ in the transient receptor potential cation channel, subfamily $M$, member 4 gene (TRPM4) at chromosomal locus 19q13.3. This mutation predicted the amino acid substitution $\mathrm{P} . \mathrm{E} 7 \mathrm{~K}$ in the TRPM4 amino terminus. TRPM4 encodes a $\mathrm{Ca}^{2+}$ activated nonselective cation (CAN) channel that belongs to the transient receptor potential melastatin ion channel family. Quantitative analysis of TRPM4 mRNA content in human cardiac tissue showed the highest expression level in Purkinje fibers. Cellular expression studies showed that the c.19G $\rightarrow$ A missense mutation attenuated deSUMOylation of the TRPM4 channel. The resulting constitutive SUMOylation of the mutant TRPM4 channel impaired endocytosis and led to elevated TRPM4 channel density at the cell surface. Our data therefore revealed a gain-of-function mechanism underlying this type of familial heart block.
\end{abstract}

\section{Introduction}

The electrical impulse of each heartbeat is propagated through the heart by direct cell-cell coupling of cardiac muscle cells and through the cardiac conduction system, composed of the sinoatrial node (the natural pacemaker [PM]), ill-defined atrial tracks, and the atrioventricular node, and thereafter the ventricular conduction system, formed by the bundle of His, the left and right bundle branches, and Purkinje fibers (1). Conduction block at the atrioventricular level or more distal (i.e., complete heart block [CHB]) is one of the most common indications for PM implantation, with 3 of 1,000 individuals in Europe receiving PMs (2). Because PM implantation has been shown to improve survival (3), conduction disease poses an important health problem. Causal loci have been mapped for single-gene diseases involving atrioventricular conduction in isolation or associated with cardiomyopathy or skeletal myopathy; for a number of diseases, the actual gene has been identified (4). One of these diseases is progressive familial heart block type I (PFHBI; also known as PFHBIB), an autosomal-dominantly inherited disease of the His-Purkinje system; a number of microsatellite markers (5) have mapped PFHBI to chromosome 19q13.3 (multipoint LOD score, 11.6, centering in a 10-cM interval around the kallikrein I [KLK1] locus; the highest 2-point LOD score for KLK1 is 6.49 with $\Theta$ of 0 ). A disease sharing phenotypic characteristics, described as isolated cardiac conduction disorder (ICCD) in a Lebanese kindred, has been mapped to the same chromosomal location (6). Furthermore, another progressive cardiac conduction disease sharing features with PFHBI is caused by mutations in a

Authorship note: Martin Kruse, Eric Schulze-Bahr, and Valerie Corfield contributed equally to this work.

Conflict of interest: The authors have declared that no conflict of interest exists. Citation for this article: J. Clin. Invest. 119:2737-2744 (2009). doi:10.1172/JCI38292. sodium channel, $S C N 5 A$, on chromosome $3(7,8)$. In PFHBI, disease progression is characterized initially by the occurrence of right bundle branch block (RBBB), followed by bifascicular block and finally CHB (9). Another conduction disease, PFHBII (also known as PFHB2), described at the same time as PFHBI, differs clinically: $\mathrm{CHB}$ occurrence is consistent with disease at an atrioventricular nodal level $(9,10)$, not with ventricular conduction disease.

In the PFHBI linkage study (5), the phenotype was based on ECG-defined RBBB, bifascicular block, or CHB with broad QRS complexes, or the presence of an implanted PM if clinical details indicating this intervention were unknown (Figure 1). Subsequent high-resolution mapping permitted identification of carriers and noncarriers based on the disease-associated haplotype (5), and the information has been used to counsel at-risk individuals about their carrier status.

Here, we further refined the genetic interval for the PFHBI disease locus and showed that a missense mutation in TRPM 4 is the cause of blunted cardiac conduction in several branches of a large Afrikaner family. TRPM4 encodes a $\mathrm{Ca}^{2+}$-activated nonselective cation (CAN) channel in in vitro expression systems (11). Cardiac CAN channel activity has been suggested to contribute to the transient inward current $\left(\mathrm{I}_{\mathrm{ti}}\right)$ initiated by Ca ${ }^{2+}$ waves. $\mathrm{I}_{\mathrm{ti}}$ was described on Purkinje fibers, atrial and ventricular cardiomyocytes, and sinoatrial node cells (12). The identity of $\mathrm{I}_{\mathrm{ti}}$ is controversial, but it appears to reflect $3 \mathrm{Ca}^{2+}$ dependent components: $\mathrm{Na}^{+} / \mathrm{Ca}^{2+}$ exchange, $\mathrm{Ca}^{2+}$-activated chloride current, and current mediated by CAN channels such as TRPM4 (previously referred to as TRPM4b; refs. 12, 13). The PFHBI-associated mutation, which results in an amino acid sequence change in the TRPM $4 \mathrm{~N}$ terminus, led to constitutive SUMOylation of TRPM4 and impaired TRPM4 endocytosis, resulting in a dominant gain of TRPM4 channel function. Our findings indicate a key role for the TRPM4 CAN channel in cardiac conduction. 
A

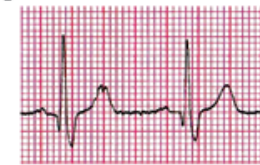

Std I

B

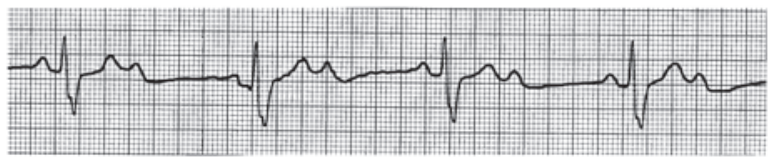

\section{Figure 1}

Cardiac phenotype of PFHBI patients. Shown are electrocardiographic examples of PFHBI. (A) Sinus rhythm with a RBBB in an 8-year-old asymptomatic boy on a standard 12-lead ECG, with leads Std I, V1, and V6 shown. (B) Shown is 2:1 atrioventricular node block (atrial rate, $76 \mathrm{bpm}$; ventricular rate, $38 \mathrm{bpm}$ ) with a broad QRS complex on Holter monitoring in a 54-year-old man who had recently become symptomatic. ECGs were recorded at a $25 \mathrm{~mm} / \mathrm{s}$ paper speed and $10 \mathrm{~mm} / \mathrm{mV}$ signal amplitude.

\section{Results}

PFHBI disease is linked to a mutation in TRPM4. We extended the clini$\mathrm{cal}$ and genetic basis of our previous mapping and linkage study in the Afrikaner pedigree with PFHBI, traced to an ancestral founder who immigrated from Portugal to South Africa in 1696 (5). Of 71 mutation carriers identified, 48 had PMs implanted. For some family members with PMs, we had access to ECGs prior to the date of PM implantation in which they still had normal atrioventricular conduction. Of the combined individuals not in need of PMs and patients with normal atrioventricular conduction prior to implantation, 19 patients had RBBB, 8 had RBBB with left anterior or left posterior hemiblock, and 7 had no ECG abnormalities (Supplemental Table 1; supplemental material available online with this article; doi:10.1172/JCI38292DS1). For the 19 patients with $\mathrm{RBBB}$, heart rates, mean P-wave duration, and mean PR interval were in the normal range (Supplemental Table 1). Atrial premature activity and extra systoles were not seen. None of the characterized patients showed a Brugada syndrome type I pattern in ECG analysis, and syncope caused by Torsades de pointes was not observed in these families. It should be noted that with bundle branch blocks, QTc may be prolonged because of late depolarization, and consequent late repolarization, of one ventricle.

With additional microsatellite markers, we narrowed the PFHBI locus to an interval between markers D19S1059 and D19S604 corresponding to a region of approximately $0.5 \mathrm{Mb}$ in chromosome 19 q13.33 with approximately 25 genes (Supplemental Figure 1 ). Of these 25 genes, 5 are expressed in cardiac tissue: $U 1$ small nuclear ribonucleoprotein (SNRP70); histidine-rich calcium-binding protein (HRC); transient receptor potential cation channel, subfamily $\mathrm{M}$, member 4 (TRPM4); transcriptional enhancer 4 (TEAD2); and potassium voltage-gated channel, Shaker-related subfamily, member7 (KCNA7) (11, 14-18). Previously, KCNA7 was excluded as a possible candidate gene (16). Here, we focused on TRPM4 (11), which was prominently expressed in human Purkinje fibers compared with septum, atrium, and right and left ventricles (Figure 2A). We sequenced the 25 exons of TRPM4 (Supplemental Table 2). DNA samples of 23 affected and 35 unaffected members of the Afrikaner pedigree with PFHBI were available for analysis. We detected exclusively in DNA samples of affected family members a heterozygous $\mathrm{G} \rightarrow$ A mutation at nucleotide 19 in exon 1 (c.19G $\rightarrow$ A; Figure $2 B$ ). The mutation generated a new recognition site for the restriction enzyme MboII, allowing for independent identification of the $\mathrm{c} .19 \mathrm{G} \rightarrow \mathrm{A}$ TRPM 4 mutation by MboII DNA digestion (Supplemental Figure 2). In 2 unaffected control populations (230 ancestry-matched, unrelated Afrikaner and 389 unrelated individuals of mixed European descent), we did not detect the 19A allele. The $\mathrm{c} .19 \mathrm{G} \rightarrow \mathrm{A}$ mutation in TRPM4 predicts the substitution of TRPM4 glutamic acid at position 7 to lysine (p.E7K) within the TRPM4 N terminus (referred to herein as TRPM4 ${ }^{\mathrm{E} 7 \mathrm{~K}}$ ). Glu7 is part of an N-terminal TRPM4 sequence motif evolutionary conserved among TRPM4 orthologs across phyla (Figure 2C). The motif is likely to play an important function in TRPM4 channel activity unrelated to conserved sequence domains previously reported to affect TRPM4 channel assembly or activity (Figure 2D and ref. 19).

The PFHBI-associated mutation increases TRPM4 current density. To understand the functional significance of the PFHBI-associated mutation, we expressed WT and mutant TRPM4 channels in HEK 293 cells and measured TRPM4 current properties in response to a variety of stimuli known to influence TRPM4 channel gating $(11,19)$. WT and mutant TRPM4 channels responded in an essentially identical manner to both change in membrane voltage and rise in intracellular $\mathrm{Ca}^{2+}$ (Figure 3, A and B). At $+80 \mathrm{mV}$, half-maximal current activation was observed, with $0.44 \pm 0.03$ and $0.40 \pm 0.04 \mu \mathrm{M} \mathrm{Ca}^{2+}$ for WT and mutant TRPM4 channels, respectively ( $n=7$ per group; Figure $3 \mathrm{~B}$ ), in agreement with data previously reported for the TRPM4 channel expressed in HEK 293 cells (11). WT and mutant TRPM4 channel exhibited essentially identical sensitivities to block by intracellular ATP in inside-out membrane patches. Bath application of $500 \mu \mathrm{M}$ adenylyl imidodiphosphate tetralithium salt (AMP-PNP), a nonhydrolizable ATP analog, inhibited WT and mutant TRPM4 channels with similar potency (TRPM4, $72.5 \% \pm 2.5 \%$ block; TRPM4 ${ }^{\mathrm{E} 7 \mathrm{~K}}, 71.3 \% \pm 3.9 \%$ block, $n=3$; Figure 3C, Supplemental Figure 3A, and ref. 20). Furthermore, application of phosphatidylinositol-4,5-bisphosphate $\left[\operatorname{PtdIns}(4,5) \mathrm{P}_{2}\right]$ to inside-out patches stimulated TRPM4 and TRPM4 4 E7K current amplitudes to a similar degree (TRPM4, 232\% $\pm 12 \%$, TRPM4 $47 \mathrm{~K}$, $238 \% \pm 15 \%, n=3$; Supplemental Figure 3, B and C). These data indicated that PtdIns $(4,5) \mathrm{P}_{2}$ sensitivity of TRPM4 ${ }^{\mathrm{E} 7 \mathrm{~K}}$ channels was unchanged compared with WT channels (21).

However, comparison of TRPM4 channel densities showed that HEK 293 cells expressing TRPM4 ${ }^{\mathrm{E} 7 \mathrm{~K}}$ consistently exhibited approximately 2 -fold TRPM4 current density $(43.8 \pm 6.3 \mathrm{pA} / \mathrm{pF}$ at $+80 \mathrm{mV}, n=13)$ compared with that of cells expressing WT channel $(18.8 \pm 3.7 \mathrm{pA} / \mathrm{pF}$ at $+80 \mathrm{mV}, n=10)$. We obtained identical results using $\mathrm{CHO}$ cells (Supplemental Figure 4). Furthermore, HEK 293 cells transfected with TRPM4 ${ }^{\text {E7K }}$ cDNA or cotransfected with equal amounts of TRPM4 and TRPM4 $4^{\mathrm{E}} \mathrm{K}$ cDNA $(43.9 \pm 5.2 \mathrm{pA} / \mathrm{pF}$ at $+80 \mathrm{mV}, n=6)$ showed essentially the same increase in current density (Figure 3D). The dominant mutational effect on TRPM4 current amplitude apparently reflects the dominant inheritance of the PFHBI phenotype.

Macroscopic current amplitude $I$ is the product of 3 parameters: single-channel conductance $(\gamma)$, open probability $\left(P_{o}\right)$, and number of channels $(N)$ expressed at the cell surface. First, we investigated in the inside-out patch-clamp configuration parameters $\gamma$ and $P_{o}$, biophysical parameters intrinsic to the channel. They were essentially 
A

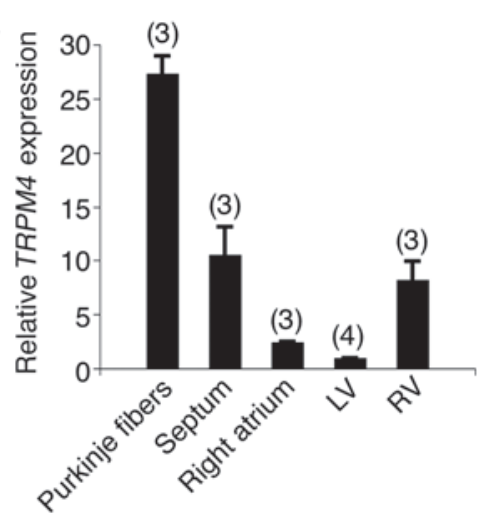

C

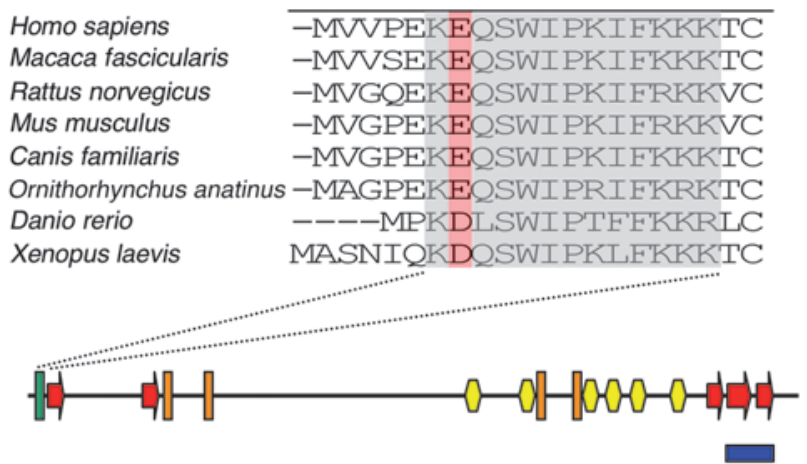

\PFHBI \СаM \WB ОTM $\square \mathrm{CCR}$

identical for WT $\left(\gamma, 19.1 \pm 0.8 \mathrm{pS} ; P_{o}, 0.25 \pm 0.09, n=8\right)$ and TRPM4 ${ }^{\mathrm{E} 7 \mathrm{~K}}$ channels $\left(\gamma, 18.8 \pm 0.4 \mathrm{pS} ; P_{o}, 0.26 \pm 0.02, n=9\right.$; Figure $\left.3, \mathrm{E}-\mathrm{G}\right)$. The data indicate that the observed mutational effect on TRPM4 current amplitude was most likely the result of a change in $N$.

The PFHBI-associated mutation increases TRPM4 channel density. We addressed this hypothesis directly and evaluated the number of WT and mutant TRPM4 channels residing in the plasma membrane. In order to evaluate $N$, we introduced a Myc tag into an extracellular site of TRPM4 between the first and second transmembrane-spanning segments, according to the predicted membrane topology of TRPM4 (Supplemental Figure 5 and ref. 19). Functional tests showed that Myc-tagged WT and mutant TRPM4 channels mediated currents indistinguishable from the untagged channel (Supplemental Figure 6, A and B). Next, we labeled nonpermeabilized HEK 293 cells expressing Myc-tagged WT or TRPM4E7K channels with FITC-coupled anti-Myc antibody and quantitated $N$ using fluorescence-activated cell sorting (FACS; Figure 4A). HEK 293 cells expressing untagged WT channel were used for background control. In agreement with our electrophysiological results, the FACS results showed approximately 2.5-fold larger $N$ for Myc-tagged TRPM4 ${ }^{\text {E7K }}$ than for WT TRPM (Figure 4, A and B).

A likely explanation for increased TRPM4 ${ }^{\mathrm{E} 7 \mathrm{~K}}$ channel density in the plasma membrane was a mutational influence on TRPM4 protein stability. To address this hypothesis, we investigated the steady-state concentration of TRPM4 WT and mutant channel protein in transiently transfected cells by Western blot and immunofluorescence microscopy. We used FLAG-tagged channels with current density similar to that of untagged controls (Supplemental Figure 6, C and D) to analyze TRPM4 protein concentra-

\section{Figure 2}

TRPM4 missense mutation in exon 1 associated with PFHBI. (A) Relative expression of TRPM4 transcripts in different tissues of nondiseased human heart was assayed by quantitative RT-PCR. TRPM4 mRNA expression levels were normalized to the level in left ventricle. Numbers of individual probes are shown in parentheses. Each experiment was done in triplicate. (B) Electropherograms show TRPM4 WT sequence and the heterozygous sequence change $\mathrm{C} .19 \mathrm{G} \rightarrow \mathrm{A}$ in the DNA of PFHBI-affected individuals. (C) Partial amino acid sequence alignment of TRPM4 N terminus among different species. Gray shading shows the conserved sequence motif; red shading highlights the glutamic acid substituted by lysine in TRPM4 associated with PFHBI. Numbering refers to the human sequence. (D) Diagram of TRPM4 topology and functional domains, with 6 membrane-spanning domains (TM) flanked by $\mathrm{N}$ - and $\mathrm{C}$-terminal cytoplasmic sequences. PFHBI, PFHBI domain; CaM, Calmodulin-binding domain; WB, Walker B ATPbinding motif; CCR, coiled-coiled region. Figure part adapted with permission from Pflügers Archiv (19).

tion in transfected cells. Western blot analysis of respective cell lysates revealed a more intense signal for FLAG-tagged TRPM4 ${ }^{\mathrm{E} 7 \mathrm{~K}}$ than for WT ( $n=3$; Figure 4E). Fluorescence image quantitation (see Methods) revealed significantly larger immunofluorescence intensity for TRPM4 $4^{\mathrm{E} T \mathrm{~K}}$ than for WT (Figure 4, C and D). Thus, the effect of the PFHBI mutation on TRPM4 channel density correlated with an elevated steady-state level of TRPM4 protein.

The PFHBI-associated mutation affects TRPM4 channel endocytosis. The observed TRPM $4^{\mathrm{E} 7 \mathrm{~K}}$ concentration increase in the plasma membrane indicated an altered balance between the anterograde and retrograde TRPM4 trafficking pathways that determine steady-state protein expression at the cell surface. Because mutations mostly obstruct protein function (22-27), we considered a default in retrograde (i.e., endocytotic) TRPM4 trafficking as a likely explanation for the PFHBI mutational effect. Dynein-based endocytotic trafficking, a common pathway in ion channel endocytosis (28-30), is effectively inhibited in cells that overexpress dynamitin $(31,32)$. Overexpression of dynamitin is an established method to disrupt the dynein motor system and to interfere with ion channel endocytosis. Thus, we investigated the effect of dynamitin overexpression on TRPM4 channel density. Cotransfection of HEK 293 cells with dynamitin and TRPM4 channel cDNAs resulted in significantly increased TRPM4 channel density $(39.3 \pm 7.9 \mathrm{pA} / \mathrm{pF}, n=9$; Figure $5 \mathrm{~A})$. Conversely, TRPM4 ${ }^{\mathrm{E} 7 \mathrm{~K}}$ current density was dynamitin insensitive $(42.7 \pm 8.7 \mathrm{pA} / \mathrm{pF}, n=7$; Figure $5 \mathrm{~A})$. We conclude that the PFHBI mutation impaired dynein-based TRPM4 channel endocytosis.

Endocytosis is the initial step in retrograde movement of membrane protein. Subsequently, internalized proteins can follow multiple routes with different outcomes. One well-recognized fate of internalized protein is targeting for proteasomal degradation (33). Therefore, we reasoned that WT TRPM4 is more sensitive to proteasomal degradation than is TRPM4 ${ }^{\mathrm{E} 7 \mathrm{~K}}$, for which endocytosis is disrupted. To test this hypothesis, we investigated the effect of the proteasome inhibitor MG132 (34) on cell surface expression of WT and mutant Myc-tagged TRPM4 channels. Quantitative FACS analysis of Myc-specific antibody-labeled transfected HEK 293 cells showed that MG132 produced a significant, approximately 3 -fold increase in channel density for WT $(310 \% \pm 30 \%, n=3)$, but not TRPM $4^{\mathrm{E} 7 \mathrm{~K}}$ (Figure $\left.5 \mathrm{~B}\right)$. This finding concurs with our observation that the endocytotic TRPM4 trafficking was impaired by PFHBI mutation, possibly by its interference with a regulatory pathway. 
A

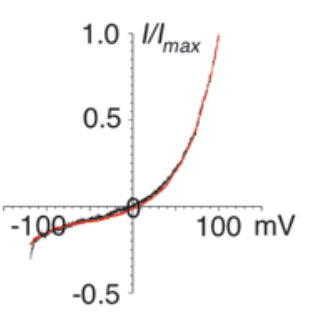

C

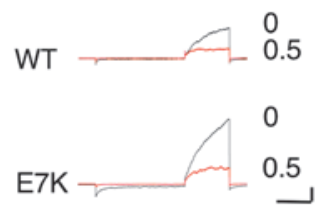

$\mathbf{E}$

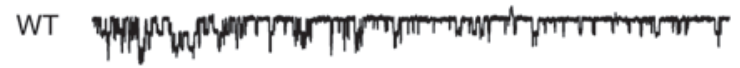
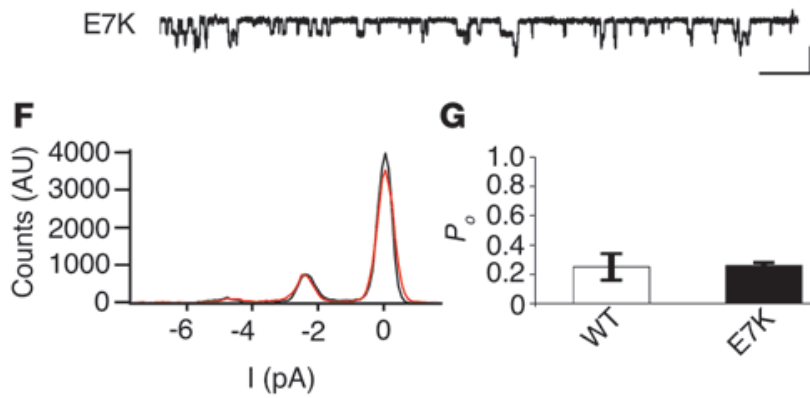

G

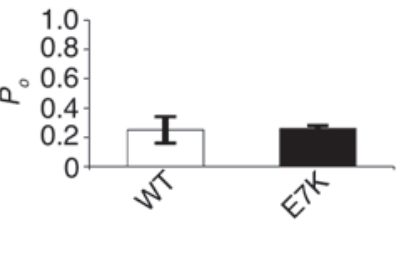

The TRPM4ETK Channel is less sensitive to SUMOylation. The regulatory pathway presumably involves posttranslational TRPM4 modification, for example, by protein phosphorylation and/or dephosphorylation. In keeping with this idea, we applied 8-Brc-AMP to TRPM4expressing HEK 293 cells to stimulate protein kinase A and then studied the effects of the phosphodiesterase inhibitor 3-isobutyl1-methylxanthine and the protein phosphatase inhibitor okadaic acid on TRPM4 current density. Both WT and TRPM4 ${ }^{\mathrm{E} 7 \mathrm{~K}}$ current density was insensitive to this treatment (Supplemental Figure 7). As an alternative posttranslational modification, one that may regulate ion channel density in the plasma membrane, we considered SUMOylation (33). First, we directly investigated SUMOylation of FLAG-tagged TRPM4. FLAG-tagged WT or mutant TRPM4 protein was pulled down with immobilized FLAG-specific antibodies from cellular lysates and immunostained with small ubiquitin-like modifier-specific (SUMO-specific) antibodies in Western blots. We found that TRMP4 protein was directly SUMOylated, whereas vector controls showed no detectable SUMOylation signal; moreover, TRPM4 ${ }^{\mathrm{E} 7 \mathrm{~K}}$ protein displayed markedly stronger immunostaining than did WT protein ( $n=3$ per group; Figure $6 \mathrm{~A})$.

Next, we investigated the effect of the SUMO ligase ubiquitinconjugating enzyme 9 (Ubc9; ref. 35) and of the SUMO protease SUMO1/sentrin specific peptidase 1 (SENP1; ref. 36) on TRPM4 current density. Coexpression of WT TRPM4 with Ubc9 produced a significant increase of TRPM4 current density $(43.3 \pm 12.7 \mathrm{pA} / \mathrm{pF}$, $n=9, P=0.04)$. Conversely, coexpression of WT TRPM4 with SENP1 decreased TRPM4 current density $(4.5 \pm 1.0 \mathrm{pA} / \mathrm{pF}, n=8)$ to background

\section{Figure 3}

Expression of human TRPM4 and TRPM4E7K in HEK 293 cells. Unless otherwise indicated, black traces denote TRPM4; red traces denote TRPM4E7K. (A) Normalized current-voltage relationship for TRPM4 and TRPM4E7K obtained from 250-ms voltage ramps measured in the whole-cell patch-clamp configuration from -120 to $+100 \mathrm{mV}$. Holding potential was $-60 \mathrm{mV}$. (B) $\mathrm{Ca}^{2+}$ dependence of TRPM4 current densities $\left(I / I_{\max }\right)$ obtained from voltage ramps measured at -80 and $+80 \mathrm{mV}$ $(n=7-16)$. $\left[\mathrm{Ca}^{2+}\right]$, intracellular $\mathrm{Ca}^{2+}$. (C) AMP-PNP block of WT TRPM4 and TRPM4E7K current. Holding potential was $0 \mathrm{mV}$. Currents, elicited by a $250-\mathrm{ms}$ pulse to $+100 \mathrm{mV}$ after a $500-\mathrm{ms}$ pulse to $-100 \mathrm{mV}$, were recorded before ( 0 ; black) and after application of $500 \mu \mathrm{M}$ AMP-PNP (0.5; red) Scale bars: $200 \mathrm{~ms}, 0.5 \mathrm{nA}$. (D) Normalized current densities $\left(I / I_{\text {norm }}\right)$ of TRPM4E7K expressed alone $(n=13)$ or in a $1: 1$ ratio with TRPM4 (WT/E7K, $n=6$ ) obtained from voltage ramps measured at +40 and $+80 \mathrm{mV} . n=16$ (WT TRPM4). ${ }^{*} P<0.05$ versus WT. (E) Single-channel currents recorded from inside-out patches at $-100 \mathrm{mV}$. Scale bars: $1 \mathrm{~s}, 5 \mathrm{pA}$. (F) Histogram plots of TRPM4 and TRPM4E7K traces shown in E. (G) $P_{o}$ of TRPM4 and TRPM4E7K channel at $+100 \mathrm{mV}(n=8-9)$.

levels measured on mock-transfected cells $(3.5 \pm 0.4 \mathrm{pA} / \mathrm{pF}, n=7$, $P=0.39)$, whereas coexpression with the inactive SENP1 mutant SENP1-C603S (37) showed no effect on WT TRPM4 current density $(21.6 \pm 3.8 \mathrm{pA} / \mathrm{pF}, n=9, P=0.79$; Figure $6 \mathrm{~B})$. In another control experiment, we investigated the effect of SENP1 protease on human ethera-go-go-related gene (HERG) channel activity, which plays an important role in ventricular action potential repolarization (38). HERG current density was insensitive to SENP1 (absence, $53.7 \pm 10.6 \mathrm{pA} / \mathrm{pF}$, $n=6$; presence, $54.8 \pm 11.2 \mathrm{pA} / \mathrm{pF}, n=7)$. In contrast to WT TRPM4, coexpression of TRPM4 ${ }^{\mathrm{E} 7 \mathrm{~K}}$ with Ubc9 $(63.3 \pm 7.1 \mathrm{pA} / \mathrm{pF}, n=17, P=0.46)$ or SENP1 (64.5 $\pm 17.2 \mathrm{pA} / \mathrm{pF}, n=6, P=0.65$; Figure 6B) neither significantly increased nor significantly decreased current density. Quantitative FACS analyses in which we investigated surface expression of Myc-tagged TRPM4 ${ }^{\mathrm{E} 7 \mathrm{~K}}$ after coexpression with Ubc9 or SENP1 yielded similar results (Figure 6C). These results imply that deSUMOylation serves as a signal for TRPM4 internalization and that, accordingly, constitutive SUMOylation protects TRPM4 ${ }^{\mathrm{E} 7 \mathrm{~K}}$ against endocytosis and proteasomal degradation.

\section{Discussion}

Here, we have identified a TRPM4 mutation in as the cause for an autosomal-dominant form of PFHBI in an Afrikaner pedigree, one of the largest reported families with chromosomal assignment (5). The mutation, TRPM4E7K, caused a dominant TRPM4 gain of function. TRPM4 ${ }^{\mathrm{E} 7 \mathrm{~K}}$ correlated with elevated density of the TRPM4 channel in the plasma membrane because of attenuated channel deSUMOylation, a mechanism in the etiology of cardiac channelopathies $(22,39)$ that was, to our knowledge, previously unknown. Gain-of-function mutations in ion channels associated with cardiac arrhythmias are relatively rare (40-42). Ion channel mutations mostly lead to loss of channel function by disrupting subunit assembly, anterograde trafficking, and/or ion channel gating properties (22-27). The TRPM4 ${ }^{\mathrm{E} 7 \mathrm{~K}}$ channel, however, displayed normal gating properties - for example, $\gamma, P_{o}$, and regulation by intracellular $\mathrm{Ca}^{2+}$, PtdIns $(4,5) \mathrm{P}_{2}$, or ATP - but increased expression at the cell surface as a result of a default in TRPM4 endocytosis. PFHBI (5) and ICCD (6) have been mapped on the same chromosomal locus and have a similar cardiac pathophysiology. It will be interesting to see whether ICCD is also associated with TRPM4 mutations increasing TRPM4 channel density at the cell surface. 
A
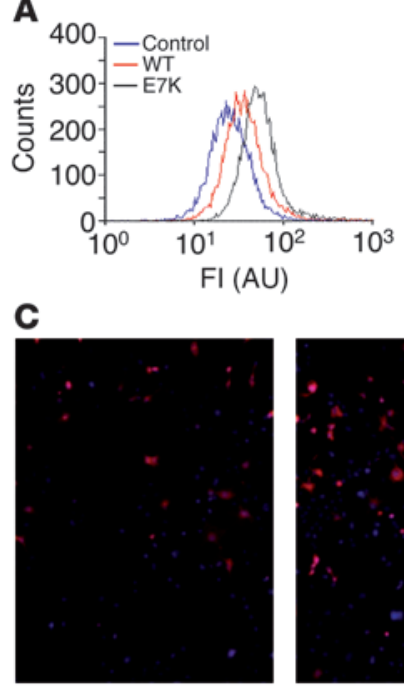

WT

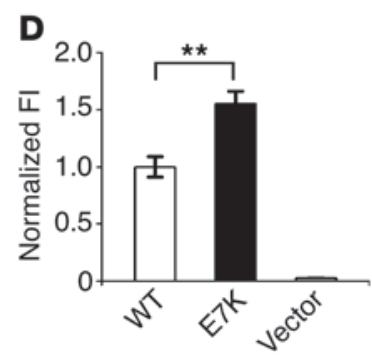

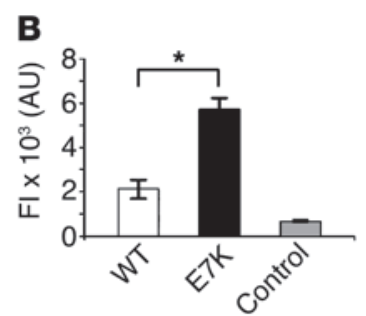

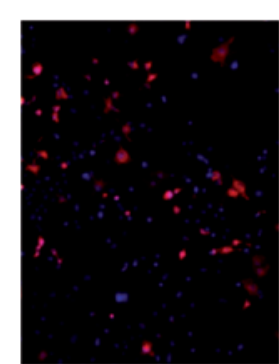

E7K

\section{$\mathbf{E}$}

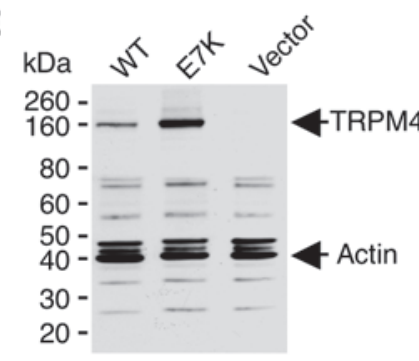

It has become apparent that SUMO and ubiquitin pathways communicate and jointly affect the properties of common substrate proteins, often having an antagonistic relationship (33). Our data showed that the expression of WT TRPM4 at the cell surface was sensitive to deSUMOylation by SENP1. In contrast, expression of TRPM4 ${ }^{\mathrm{E} 7 \mathrm{~K}}$ at the cell surface was insensitive to deSUMOylation. We therefore conclude that constitutive SUMOylation of TRPM $4^{\mathrm{E7K}}$ is responsible for the elevated expression of the channel at the cell surface. It is possible that SUMOylation of TRPM4 interferes with TRPM4 ubiquitination, an important means to target protein for internalization and proteasomal degradation, and thereby protects TRPM4 ${ }^{\mathrm{E} 7 \mathrm{~K}}$ against internalization and proteasomal degradation. Intriguingly, disruption of ubiquitin-dependent internalization has been previously demonstrated for a mutation within the gene encoding the $\mathrm{ENaC}$ channel; this mutation is associated with Liddle syndrome, a dominant hereditary form of hypertension (30).

TRPM4 and TRPM5 channels are unique candidates for the CAN channel. They are equally permeable to $\mathrm{Na}^{+}$and $\mathrm{K}^{+}$, but, in contrast to all other TRP channels, they are not permeable to $\mathrm{Ca}^{2+}(11,43)$. TRPM4 channel properties resemble those of the native cardiac CAN channel NSC $_{\mathrm{Ca}}(13,19,44)$. The importance of CAN channel activity and its contribution to the $\mathrm{Ca}^{2+}$-dependent $\mathrm{I}_{\mathrm{ti}}$ in the heart has been discussed for some time, but the physiological role in cardiac function is not well understood $(13,19,44-47)$. Our data offer a direct approach toward the physiological role of the CAN channel TRPM4. Here we showed that TRPM4 was prominently expressed in Purkinje fibers and played an important role, which we believe to be previously unrecognized, in the human cardiac conduction system. It is likely that elevated expression of the TRPM4E7K channel

\section{Figure 4}

Analysis of TRPM4 and TRPM4E7K protein density. (A) FACS fluorogram of nonpermeabilized cells expressing untagged TRPM4 control, Myc-tagged TRPM4, or Myc-tagged TRPM4E7K labeled with FITC-conjugated Myc-specific antibody. FI, fluorescence intensity. (B) FACS analysis of fluorograms in A. $n=9$ per group. (C-E) Cells expressing empty vector control (Vector), FLAG-tagged TRPM4, or FLAG-tagged TRPM4E7K were fixed and permeabilized 48 hours after transfection. (C) Cells were stained with mouse FLAG-specific primary antibody and anti-mouse goat Alexa Fluor 546-coupled secondary antibody. Nuclei were stained with DAPI. Original magnification, $\times 20$. (D) Immunofluorescence intensity of FLAG-tagged TRPM4E7K normalized to that of FLAG-tagged TRPM4-expressing cells $(n=60$ per group). (E) SDS-PAGE of C-terminally FLAG-tagged TRPM4 and TRPM4E7K protein in cell lysates followed by Western blotting. Membranes were stained with mouse FLAG-specific antibody and simultaneously with rabbit actin-specific antibody for loading control. ${ }^{\star} P<0.05,{ }^{* \star} P<0.01$.

increases membrane leak conductance, disabling action potential propagation down the Purkinje fibers. The hypothesis is consistent with the occurrence of broadened QRS complex in the patient's ECG, and could also explain the PFHBI phenotype of RBBB, bifascicular block, and heart conduction block. However, the progressive loss of ventricular conduction ability in PHFBI remains to be explored. Tentatively, chronic increase in leak conductance causes cell death, which may explain the replacement fibrosis observed postmortem in the His-Purkinje fiber system of a PFHBI patient (P. Brink, unpublished observation).

In other forms of inherited cardiac conduction disorders, mutations within cardiac $\mathrm{Na}^{+}$or $\mathrm{Ca}^{2+}$ channel genes have been identified (48-51). They are associated with loss of ion channel function as well as with reduction in depolarizing inward currents and driving force for myocellular $\mathrm{Ca}^{2+}$ entry. It has been proposed that $\mathrm{Ca}^{2+}$-sensitive transient TRPM4-mediated inward current causes membrane depolarization and thereby limits the driving force for $\mathrm{Ca}^{2+}$ entry in excitable cells $(19,44,47)$. Whether increased cardiac TRPM4 channel activity blunts cardiac conduction because of its effects on membrane depolarization and $\mathrm{Ca}^{2+}$ influx needs to be explored. Furthermore, increased TRPM4 channel activity may also influence activity of other ion channels, for example, recovery of voltage-gated ion channels from inactivation and activation of hyperpolarization-activated cation channels. The identification of TRPM $4^{\mathrm{E} 7 \mathrm{~K}}$ in patients with PFHBI paves the way for further characterization of the pathophysiology of conduction disease and may ultimately lead to less invasive therapy than the currently approach of timely PM implantation. The nature of the mutation also suggests that modulation of TRPM4 activity using $\mathrm{Ca}^{2+}$ or $\mathrm{K}^{+}$ channel inhibitors could present an avenue for treatment.

\section{Methods}

Clinical evaluation and genetic mapping. The clinical status of members of the Afrikaner pedigree was ascertained at Tygerberg Hospital, University of Stellenbosch, and phenotypically characterized as previously published (5, 9). Clinical and genetic studies were carried out according to the institutional review boards of University of Stellenbosch and the Ethikkommission der Ärztekammer Westfalen-Lippe und der Westfälischen WilhelmsUniversität Münster. Informed consent was given by all participating individuals. Detailed clinical and family histories, including cardiac symptoms, device implantation, and standard 12-lead ECGs, were obtained. Genomic DNA was isolated from venous blood samples, and 25 TRPM4 

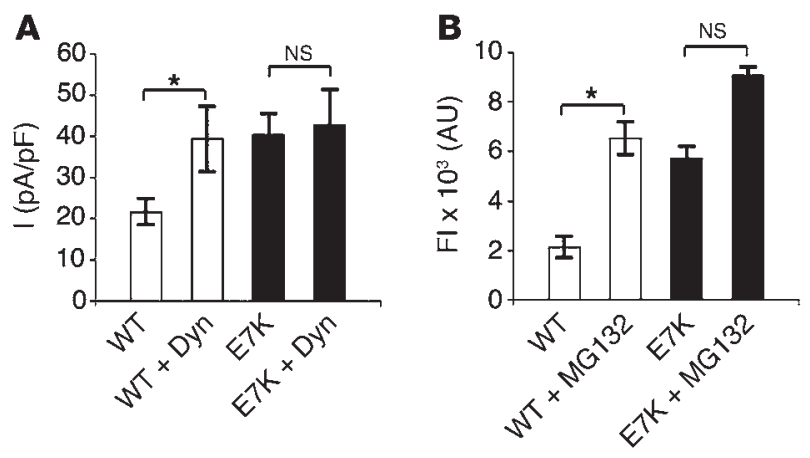

Figure 5

Posttranslational regulation of TRPM4 and TRPM4E7K channel density. (A) TRPM4 and TRPM4E7K channel, expressed together with or without dynamitin (Dyn) for 18 hours in HEK 293 cells. Current densities were obtained at $+80 \mathrm{mV}$ as described in Figure 3A. $n=7$ (E7K + Dyn); 9 (WT and WT + Dyn); 10 (E7K). (B) HEK 293 cells expressing Myctagged TRPM4 or TRPM4E7K channel were incubated with or without $10 \mu \mathrm{M}$ MG132. FACS analysis was performed as in Figure 4A. $n=3-9 .{ }^{*} P<0.05$.

exons were PCR amplified using oligonucleotides located 60-100 bp from the splice sites (Supplemental Table 2). Unrelated healthy individuals (389 mixed European descent, 230 Afrikaner) served as controls for the observed genetic variation.

Cloning of expression plasmids. For heterologous expression in tissue culture cells, the following cDNAs were cloned into pcDNA3 vector: human TRPM4 (provided by A. Guse, University Hospital Hamburg-Eppendorf, Hamburg, Germany; ref. 52), HERG (generated in our laboratory), dynamitin (provided by R. Vallee, Columbia University, New York, New York, USA; ref. 53), SENP1 (provided by P. O'Hare, Marie Curie Research Institute, Oxted, United Kingdom; ref. 36), and Ubc9 (provided by S. Müller, MaxPlanck-Institute of Biochemistry, Munich, Germany; ref. 35). TRPM4E7K cDNA was obtained by in vitro mutagenesis. For detection in Western blot or immunofluorescence experiments, $\mathrm{N}$-terminal (after Met 1) or C-terminal (after Asp 1214) FLAG tags, respectively, were inserted into the coding sequence by PCR. For quantitative FACS analysis, a Myc-Tag was inserted after Leu 722; the fluorescence image quantitation program ImageJ (http://rsbweb.nih.gov/ij/) was used for quantitative analysis of transfected cells immunostained with anti-FLAG antibodies. Plasmid sequences were verified by sequence analysis.

Quantitative RT-PCR. Heart tissue samples were a gift of U. Ravens and E. Wettwer (Technische Universität Dresden, Dresden, Germany). Tissue samples were prepared from nondiseased human hearts technically unusable for transplantation. All experimental protocols were approved by the Ethical Review Board of the University Hospital of Dresden. Total RNA

\section{Figure 6}

Sensitivity of TRPM4 current density to SUMOylation. (A) C-terminally FLAG-tagged TRPM4 or TRPM4E7K protein pulled down on beads coated with FLAG-specific antibodies were subjected to SDS-PAGE and Western blotting. Blots were stained with rabbit SUMO-1-specific primary antibody and anti-mouse rabbit horseradish peroxidase-coupled secondary antibody. Arrow indicates SUMOylated TRPM4. (B) Same experiment as in Figure 5A, but TRPM4 channel was coexpressed with Ubc9, SENP1, or the inactive mutant SENP1-C603S (SENP1*). $n=6-13$. (C) Myc-tagged TRPM4E7K was expressed in HEK 293 cells alone or together with Ubc9 or SENP1. At 24 hours after transfection, the surface density of Myc-tagged TRPM4E7K channel was assessed by FACS analysis, as described in Methods. $n=3$ per group. ${ }^{*} P<0.05$.

was extracted from heart tissue samples using TRIzOL (Invitrogen) according to the manufacturer's instructions. The RNA was reverse transcribed into cDNA with Superscript II RT (Invitrogen) and subjected to real-time PCR. TRPM4 expression was monitored using a TaqMan Assay for TRPM4 (Hs00214167_m1; Applied Biosystems Inc.). For relative expression analysis, a RPLPO TaqMan Assay was used (4333761T; Applied Biosystems Inc.). Analysis was carried out as published previously (54).

Cell culture. HEK 293, CHO, and African green monkey (COS-7) cells were cultured at $37^{\circ} \mathrm{C}$ and $5 \% \mathrm{CO}_{2}$ in DMEM (HEK 293 and COS-7) or MEM- $\alpha$ medium (CHO) supplemented with $10 \%$ FBS (Invitrogen) and $2 \mathrm{mM}$ glutamine. Plasmid DNA was transfected into HEK 293, COS-7, and $\mathrm{CHO}$ cells with Lipofectamine 2000 (Invitrogen) according to the manufacturer's specifications.

FACS analysis. HEK 293 cells were transiently transfected with plasmids encoding for TRPM4 control, Myc-tagged TRPM4, or Myc-tagged TRPM4 ${ }^{\text {E7K }}$ channels. At 24 hours after transfection, cells were harvested by Accutase (PAA Laboratories $\mathrm{GmbH}$ ), washed once with PBS, and resuspended at a density of $1 \times 10^{6}$ cells $/ \mathrm{ml}$ in FACS buffer (PBS plus $0.5 \%$ BSA and $0.02 \%$ $\mathrm{NaN}_{3}$ ). We stained $1 \times 10^{5}$ cells with FITC-coupled Myc-specific antibody (Sigma-Aldrich) at a dilution of 1:50 for 30 minutes at $4^{\circ} \mathrm{C}$. Subsequently, cells were washed with $1 \mathrm{ml}$ FACS buffer, resuspended in $400 \mu \mathrm{l}$ FACS buffer, and directly analyzed for FITC fluorescence.

Western blot. Transiently transfected COS-7 cells expressing TRPM4 were harvested 24-48 hours after transfection. Cells were pelleted by centrifugation for 3 minutes at $300 \mathrm{~g}$. Pellets were washed twice in PBS and resuspended in $1 \mathrm{ml} \mathrm{H}_{2} \mathrm{O}$. Cells were lysed by freeze-thaw cycles in liquid nitrogen and $37^{\circ} \mathrm{C}_{2} \mathrm{O} 3$ times before the nuclear fraction was pelleted by centrifugation for 3 minutes at $2,000 \mathrm{~g}\left(4^{\circ} \mathrm{C}\right)$. Supernatant was removed, and the membrane-enriched fraction was pelleted for 20 minutes at $18,000 \mathrm{~g}$ $\left(4^{\circ} \mathrm{C}\right)$, resuspended in 30-50 $\mu \mathrm{l} \mathrm{HEPES}-l y s i s$ buffer $(150 \mathrm{mM} \mathrm{NaCl}, 50 \mathrm{mM}$ HEPES, and $0.5 \%$ Triton X-100, pH 7.4), and subjected to SDS-PAGE. Proteins were transferred onto PROTRAN nitrocellulose membrane (What-

A
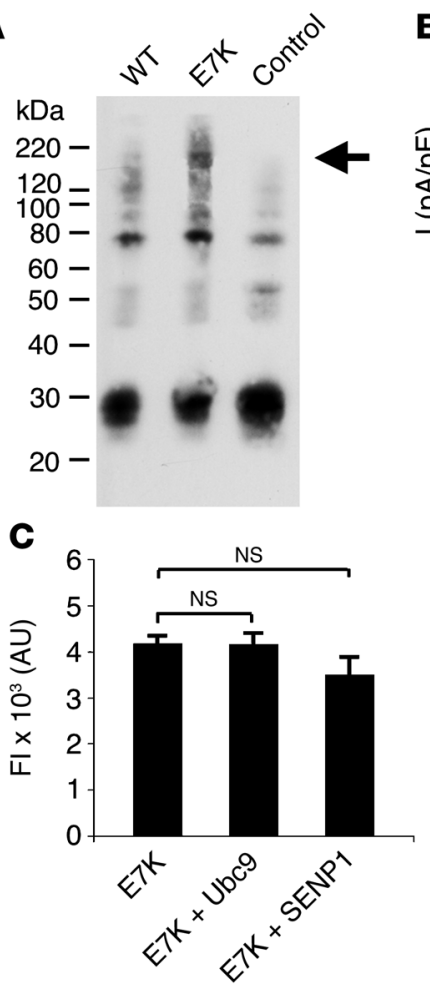

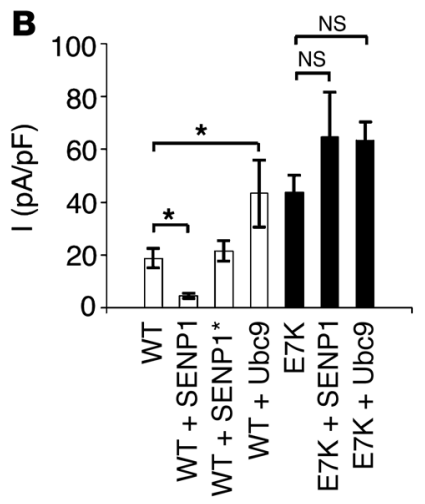


man $\mathrm{GmbH}$ ) and stained with antibodies against FLAG tag (diluted 1:500) for TRPM4 expression, SUMO-1 (diluted 1:2,000), or actin (diluted 1:2,000; all from Sigma-Aldrich).

Immunofluorescence. COS-7 cells were plated onto glass coverslips and transiently transfected with expression plasmids for TRPM4 or TRPM4 ${ }^{\mathrm{E} 7 \mathrm{~K}}$. At 48 hours after transfection, cells were fixed with $4 \%$ PFA and permeabilized with $0.2 \%$ Triton X-100. After blockade for unspecific binding with $10 \%$ horse serum, cells were stained for TRPM4 expression by mouse FLAG-specific antibody (Sigma-Aldrich) at a dilution of $1: 500$ at $22^{\circ} \mathrm{C}$ for 1 hour. After washing, cells were treated with a goat anti-mouse secondary antibody coupled to Alexa Fluor 546 (Invitrogen) for 20 minutes at $22^{\circ} \mathrm{C}$ in a 1:1,000 dilution. Stained cells were analyzed with a Fluoview FV1000 confocal microscope (Olympus GmbH).

Electrophysiology. Whole-cell, inside-out, and single-channel patch-clamp recordings were performed with an EPC9 patch-clamp amplifier (HEKA Elektronik) at a sampling rate of $20 \mathrm{kHz}$. Patch electrodes had a DC resistance between 1 and $3 \mathrm{M} \Omega$ when filled with intracellular solution for whole-cell and inside-out patches, and between 6 and $10 \mathrm{M} \Omega$ for single-channel recordings. An Ag-AgCl wire was used as a reference electrode. Capacitance and access resistance were monitored continuously, and cell membrane capacitance values were used to calculate current densities. For whole-cell recordings on HEK 293 and CHO cells, the bath solution used was buffer A $(156 \mathrm{mM} \mathrm{NaCl}$, $5 \mathrm{mM} \mathrm{CaCl}_{2}, 10 \mathrm{mM}$ glucose, and $10 \mathrm{mM}$ HEPES) at $\mathrm{pH}$ 7.2. The pipette solution used was buffer B (156 mM CsCl, $1 \mathrm{mM} \mathrm{MgCl} 2,10 \mathrm{mM}$ EGTA, and $10 \mathrm{mM}$ HEPES) at $\mathrm{pH}$ 7.4. Free $\mathrm{Ca}^{2+}$ in the pipette solution was 0.02 and $7.4 \mu \mathrm{M}$ by addition of $\mathrm{CaCl}_{2}$. $\mathrm{Ca}^{2+}$ concentrations were measured with the $\mathrm{Ca}^{2+}$-sensitive dye Fluo4FF (Invitrogen). Holding potential was $-60 \mathrm{mV}$, and current traces were elicited by voltage ramps for $250 \mathrm{~ms}$ from -120 to $+100 \mathrm{mV}$ (52). Inside-out patch-clamp recordings, for determination of TRPM4 channel block induced by AMP-PNP (Sigma-Aldrich), were performed with buffer $\mathrm{A}$ at $\mathrm{pH} 7.4$ as bath and pipette solution. For application of AMP-PNP, cells were superfused with buffer B plus $0.3 \mathrm{mM} \mathrm{CaCl}_{2}$ at $\mathrm{pH} 7.2$, with and without $500 \mu \mathrm{M}$ AMP-PNP (20). Holding potential was $0 \mathrm{mV}$; channels were activated with a 250 -ms pulse to $+100 \mathrm{mV}$ after a 500 -ms pulse to $-100 \mathrm{mV}$ (20). For single-channel recordings on $\mathrm{CHO}$ cells, bath solution contained $140 \mathrm{mM} \mathrm{NaCl}, 4.8 \mathrm{mM} \mathrm{KCl}, 1.2 \mathrm{mM} \mathrm{MgCl}_{2}, 10 \mathrm{mM}$ glucose, and $10 \mathrm{mM}$ HEPES ( $\mathrm{pH}$ 7.4). The pipette solution used was buffer C (145 mM $\mathrm{NaCl}, 1 \mathrm{mM} \mathrm{CaCl}_{2}, 1.2 \mathrm{mM} \mathrm{MgCl}_{2}, 10 \mathrm{mM}$ glucose, and $10 \mathrm{mM}$ HEPES) at $\mathrm{pH}$ 7.4. For activation of TRPM4 channels, patches were superfused with buffer $\mathrm{C}$ at $\mathrm{pH} 7.2$ (47). Bath solution for recordings of HERG channels in HEK 293 cells contained $150 \mathrm{mM} \mathrm{NaCl}, 1.8 \mathrm{mM} \mathrm{CaCl}_{2}, 5 \mathrm{mM}$ glucose, $4 \mathrm{mM}$
$\mathrm{KCl}, 1 \mathrm{mM} \mathrm{MgCl}_{2}$, and $10 \mathrm{mM}$ HEPES (pH 7.4). Pipette solution was composed of $126 \mathrm{mM} \mathrm{KCl}, 4 \mathrm{mM} \mathrm{Mg-ATP}, 2 \mathrm{mM} \mathrm{MgSO}_{4}, 5 \mathrm{mM}$ EGTA, $0.5 \mathrm{mM}$ $\mathrm{CaCl}_{2}$, and $25 \mathrm{mM} \mathrm{HEPES} \mathrm{(} \mathrm{pH}$ 7.3). We measured $\mathrm{K}$ currents by 3.5 -second depolarizing test pulses from a holding potential of $-80 \mathrm{mV}$ to test potentials between -50 and $+50 \mathrm{mV}$, followed by repolarization to $-40 \mathrm{mV}(55)$. Recordings were carried out at room temperature $\left(22^{\circ} \mathrm{C}-25^{\circ} \mathrm{C}\right)$.

Accession numbers. GenBank accession numbers are as follows: Homo sapiens TRPM4 cDNA, NM_017636; Homo sapiens TRPM4, NP_060106; Macaca fascicularis TRPM4, BAC41778; Rattus norvegicus TRPM4, XP_574447; Mus musculus TRPM4, AAH96475; Canis familiaris TRPM4, XP_541500; Ornithorbynchus anatinus TRPM4, XP_001508557; Danio rerio TRPM4, XP_001339981; Xenopus laevis TRPM4, AAH72955.

Statistics. All data are presented as mean \pm SEM. To test for statistical significance, unpaired 2-tailed Student's $t$ test was used. A $P$ value less than 0.05 was considered significant.

\section{Acknowledgments}

The authors thank the participating families; A. Goosen for logistical support; L. Hofmeyer for updating ECG information; U. Ravens and E. Wettwer for providing heart tissue samples; G. Sachse for critical discussion; K. Steinbach for assistance with FACS analysis; and M. Raetz, K. Hergarten, M. Keuthen, and E. Deravanessian for excellent technical and logistical help. This study was supported by grants from the South African Medical Research Council and the Harry and Doris Crossley Foundation to P. Brink and V. Corfield. O. Pongs and E. Schulze-Bahr are supported by Deutsche Forschungsgemeinschaft grants Po 137/372 (FOR 604), Po 137/39-1 (FOR 885), and DFG 653/Ki13-1 and 13-2; by the Fonds der Chemischen Industrie, and by the Interdisziplinäres Zentrum für Klinische Forschung (Medical Faculty of the University of Münster).

Received for publication December 9, 2008, and accepted in revised form June 10, 2009.

Address correspondence to: Olaf Pongs, Falkenried 94, 20251 Hamburg, Germany. Phone: 49-40-7410-55082; Fax: 49-40-741055102; E-mail: morin@zmnh.uni-hamburg.de.

Alf Beckmann's present address is: MVZ Dortmund, Dortmund, Germany.
1. Josephson, M.E., Zimetbaum, P., Marchlinski, F.E., and Buxton, A.E. 1998. The bradyarrhythmias: disorders of sinus node function and AV conduction disturbances. In Harrison's principles of internal medicine. A.S. Fauci, et al., editors. McGraw-Hill. New York, New York, USA. 1253-1261.

2. Ector, H., et al. 2000. The registry of the European Working Group on Cardiac Pacing (EWGCP). A working group of the European Society of Cardiology. Europace. 2:251-255.

3. Epstein, A.E., et al. 2008. ACC/AHA/HRS 2008 Guidelines for Device-Based Therapy of Cardiac Rhythm Abnormalities: a report of the American College of Cardiology/American Heart Association Task Force on Practice Guidelines (Writing Committee to Revise the ACC/AHA/NASPE 2002 Guideline Update for Implantation of Cardiac Pacemakers and Antiarrhythmia Devices): developed in collaboration with the American Association for Thoracic Surgery and Society of Thoracic Surgeons. Circulation 117:e350-e408.

4. Benson, D.W. 2004. Genetics of atrioventricular conduction disease in humans. Anat. Rec. A. Discov.
Mol. Cell. Evol. Biol. 280:934-939.

5. Brink, P.A., et al. 1995. Gene for progressive familial heart block type I maps to chromosome $19 \mathrm{q} 13$. Circulation. 91:1633-1640.

6. de Meeus, A., et al. 1995. An isolated cardiac conduction disease maps to chromosome 19q. Circ. Res. 77:735-740.

7. Schott, J.J., et al. 1999. Cardiac conduction defects associate with mutations in SCN5A. Nat. Genet. 23:20-21.

8. Probst, V., et al. 2003. Haploinsufficiency in combination with aging causes SCN5A-linked hereditary Lenegre disease. J. Am. Coll. Cardiol. 41:643-652.

9. Brink, A.J., and Torrington, M. 1977. Progressive familial heart block - two types. S. Afr. Med. J. 52:53-59.

10. Fernandez, P., Moolman-Smook, J., Brink, P., and Corfield, V. 2005. A gene locus for progressive familial heart block type II (PFHBII) maps to chromosome 1q32.2-q32.3. Hum. Genet. 118:133-137.

11. Launay, P., et al. 2002. TRPM4 is a Ca2+-activated nonselective cation channel mediating cell membrane depolarization. Cell. 109:397-407.
12. Guinamard, R., Demion, M., Chatelier, A., and Bois, P. 2006. Calcium-activated nonselective cation channels in mammalian cardiomyocytes. Trends Cardiovasc. Med. 16:245-250.

13. Guinamard, R., et al. 2004. Functional characterization of a $\mathrm{Ca}(2+)$-activated non-selective cation channel in human atrial cardiomyocytes. J. Physiol. 558:75-83.

14. Kaneko, K.J., Rein, T., Guo, Z.S., Latham, K., and DePamphilis, M.L. 2004. DNA methylation may restrict but does not determine differential gene expression at the Sgy/Tead2 locus during mouse development. Mol. Cell. Biol. 24:1968-1982.

15. Gregory, K.N., et al. 2006. Histidine-rich Ca binding protein: a regulator of sarcoplasmic reticulum calcium sequestration and cardiac function. J. Mol. Cell. Cardiol. 40:653-665.

16. Bardien-Kruger, S., et al. 2002. Characterisation of the human voltage-gated potassium channel gene, KCNA7, a candidate gene for inherited cardiac disorders, and its exclusion as cause of progressive familial heart block I (PFHBI). Eur. J. Hum. Genet. 10:36-43.

17. Heidecker, B., et al. 2008. Transcriptomic biomark- 
ers for individual risk assessment in new-onset heart failure. Circulation. 118:238-246.

18. Nilius, B., et al. 2003. Voltage dependence of the Ca2+-activated cation channel TRPM4. J. Biol. Chem. 278:30813-30820.

19. Nilius, B., and Vennekens, R. 2006. From cardiac cation channels to the molecular dissection of the transient receptor potential channel TRPM4 Pflugers Arch. 453:313-321.

20. Nilius, B., Prenen, J., Voets, T., and Droogmans, G. 2004. Intracellular nucleotides and polyamines inhibit the $\mathrm{Ca} 2+$-activated cation channel TRPM4b. Pflugers Arch. 448:70-75.

21. Voets, T., and Nilius, B. 2007. Modulation of TRPs by PIPs. J. Physiol. 582:939-944.

22. Knollmann, B.C., and Roden, D.M. 2008. A genetic framework for improving arrhythmia therapy. Nature. 451:929-936.

23. Lees-Miller, J.P., Duan, Y., Teng, G.Q., Thorstad, K., and Duff, H.J. 2000. Novel gain-of-function mechanism in $\mathrm{K}(+)$ channel-related long-QT syndrome: altered gating and selectivity in the HERG1 N629D mutant. Circ. Res. 86:507-513.

24. Fredj, S., Lindegger, N., Sampson, K.J., Carmeliet, P., and Kass, R.S. 2006. Altered Na+ channels promote pause-induced spontaneous diastolic activity in long QT syndrome type 3 myocytes. Circ. Res. 99:1225-1232

25. Furutani, M., et al. 1999. Novel mechanism associated with an inherited cardiac arrhythmia: defective protein trafficking by the mutant HERG (G601S) potassium channel. Circulation. 99:2290-2294.

26. Vennekens, R., et al. 2007. Increased IgE-dependent mast cell activation and anaphylactic responses in mice lacking the calcium-activated nonselective cation channel TRPM4. Nat. Immunol. 8:312-320.

27. Kass, R.S. 2005. The channelopathies: novel insights into molecular and genetic mechanisms of human disease. J. Clin. Invest. 115:1986-1989.

28. Jugloff, D.G., Khanna, R., Schlichter, L.C., and Jones, O.T. 2000. Internalization of the Kv1.4 potassium channel is suppressed by clustering interactions with PSD-95. J. Biol. Chem. 275:1357-1364.

29. Choi, W.S., et al. 2005. Kv1.5 surface expression is modulated by retrograde trafficking of newly endocytosed channels by the dynein motor. Circ. Res. 97:363-371.
30. Knight, K.K., Olson, D.R., Zhou, R., and Snyder, P.M. 2006. Liddle's syndrome mutations increase $\mathrm{Na}+$ transport through dual effects on epithelial $\mathrm{Na}^{+}$ channel surface expression and proteolytic cleavage. Proc. Natl. Acad. Sci. U. S. A. 103:2805-2808.

31. Melkonian, K.A., Maier, K.C., Godfrey, J.E., Rodgers, M., and Schroer, T.A. 2007. Mechanism of dynamitin-mediated disruption of dynactin. J. Biol. Chem. 282:19355-19364.

32. Burkhardt, J.K., Echeverri, C.J., Nilsson, T., and Vallee, R.B. 1997. Overexpression of the dynamitin (p50) subunit of the dynactin complex disrupts dynein-dependent maintenance of membrane organelle distribution. J. Cell Biol. 139:469-484.

33. Ulrich, H.D. 2005. Mutual interactions between the SUMO and ubiquitin systems: a plea of no contest. Trends Cell Biol. 15:525-532.

34. Lee, D.H., and Goldberg, A.L. 1996. Selective inhibitors of the proteasome-dependent and vacuolar pathways of protein degradation in Saccharomyces cerevisiae. J. Biol. Chem. 271:27280-27284.

35. Schmidt, D., and Muller, S. 2002. Members of the PIAS family act as SUMO ligases for c-Jun and p53 and repress p53 activity. Proc. Natl. Acad. Sci.U. S. A. 99:2872-2877.

36. Bailey, D., and O'Hare, P. 2002. Herpes simplex virus 1 ICP0 co-localizes with a SUMO-specific protease. J. Gen. Virol. 83:2951-2964.

37. Xu, Z., et al. 2006. Crystal structure of the SENP1 mutant C603S-SUMO complex reveals the hydrolytic mechanism of SUMO-specific protease. Biochem. J. 398:345-352.

38. Sanguinetti, M.C., and Tristani-Firouzi, M. 2006 hERG potassium channels and cardiac arrhythmia. Nature. 440:463-469.

39. Fuster, V., et al. 2001. Hurst's The heart. 12th edition. McGraw-Hill. New York, New York, USA. 2200 pp.

40. Modell, S.M., and Lehmann, M.H. 2006. The long QT syndrome family of cardiac ion channelopathies: a HuGE review. Genet. Med. 8:143-155.

41. Priori, S.G., et al. 2005. A novel form of short QT syndrome (SQT3) is caused by a mutation in the KCNJ2 gene. Circ. Res. 96:800-807.

42. Niu, D.M., et al. 2006. A common SCN5A polymorphism attenuates a severe cardiac phenotype caused by a nonsense SCN5A mutation in a Chinese family with an inherited cardiac conduction defect. J. Med. Genet. 43:817-821.

43. Nilius, B., et al. 2005. Regulation of the Ca2+ sensitivity of the nonselective cation channel TRPM4. J. Biol. Chem. 280:6423-6433.

44. Colquhoun, D., Neher, E., Reuter, H., and Stevens, C.F. 1981. Inward current channels activated by intracellular Ca in cultured cardiac cells. Nature. 294:752-754.

45. Simard, J.M., Tarasov, K.V., and Gerzanich, V. 2007. Non-selective cation channels, transient receptor potential channels and ischemic stroke. Biochim. Biophys. Acta. 1772:947-957.

46. Prawitt, D., et al. 2003. TRPM5 is a transient Ca2+activated cation channel responding to rapid changes in [Ca2+]i. Proc. Natl. Acad. Sci. U. S. A. 100:15166-15171.

47. Demion, M., Bois, P., Launay, P., and Guinamard, R 2007. TRPM4, a Ca2+-activated nonselective cation channel in mouse sino-atrial node cells. Cardiovasc. Res. 73:531-538.

48. Tan, H.L., et al. 2001. A sodium-channel mutation causes isolated cardiac conduction disease. Nature. 409:1043-1047.

49. Hu, D., et al. 2007. Novel mutation in the SCN5A gene associated with arrhythmic storm development during acute myocardial infarction. Heart Rhythm. 4:1072-1080.

50. Watanabe, H., et al. 2008. Sodium channel beta1 subunit mutations associated with Brugada syndrome and cardiac conduction disease in humans. J. Clin. Invest. 118:2260-2268.

51. Napolitano, C., Rivolta, I., and Priori, S.G. 2003. Cardiac sodium channel diseases. Clin. Chem. Lab. Med. 41:439-444.

52. Fliegert, R., et al. 2007. Modulation of Ca2+ entry and plasma membrane potential by human TRPM4b. FEBS J. 274:704-713.

53. Ahmad, F.J., Echeverri, C.J., Vallee, R.B., and Baas, P.W. 1998. Cytoplasmic dynein and dynactin are required for the transport of microtubules into the axon. J. Cell Biol. 140:391-401.

54. Gaborit, N., et al. 2007. Regional and tissue specific transcript signatures of ion channel genes in the non-diseased human heart. J. Physiol. 582:675-693.

55. Choe, C.U., et al. 2006. C-terminal HERG (LQT2) mutations disrupt IKr channel regulation through 14-3-3epsilon. Hum. Mol. Genet. 15:2888-2902. 